\title{
Assessment of medication adherence and factors contributing to non-adherence to calcium and vitamin D as mainstay in treatment and prophylaxis of osteoporosis.
}

MOUSTAFA, R., JASSIM, Z., AZIZ, H.A., KHUDAIR, I. and SALEM, M. 


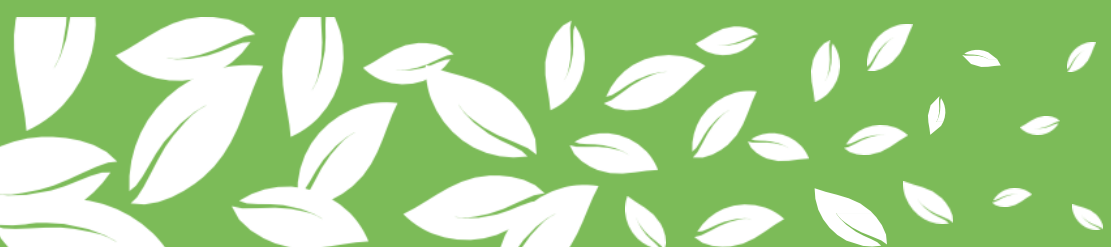

\section{Objective}

- Primary outcome: assess osteoprotic or osteopenic ambulatory patients' adherence with their calcium and vitamin D therapy.

- Secondary outcomes:

1. identify the reasons beyond theirnonadherence

2. evaluate the relationship between patients' adherence with different age groups and educational level.

\section{Method}

- Prospective- qualitative survey carried out onApril 2014.

- Inclusion criteria: aged $\geq 18$ years old, diagnosed with osteoporosis or osteopenia, taking calcium and vitamin $D$ therapy for at least one year, attended secondary care Rheumatology clinic in a teaching hospital, and willing to participate in the study.

- Modified Standardized compliance questionnaires (Morisky 8-Item Medication Adherence Questionnaire) were carrying out by clinical pharmacist in the assessment room with each patients .

- Data analyzed using descriptive \& inferentialanalyses.

\section{Results}

- There were 543 patients met the inclusion criteria, where 184 patients were taking calcium alone $(34 \%), 190$ patients taking vitamin D alone (35\%), and 169 patients taking both calcium and vitamin D (31\%).

- Age group (40-59 years) was dominant in this study (41.5\%).

- A round half of the patient's education level was college or higher.

- There was no statistically significant difference between calcium and vitaminD group in terms of adherence score $(p=0.175)$.

Figure 1. Percentage of AdherenceScore for Calcium \& Vitamin D

\section{2}

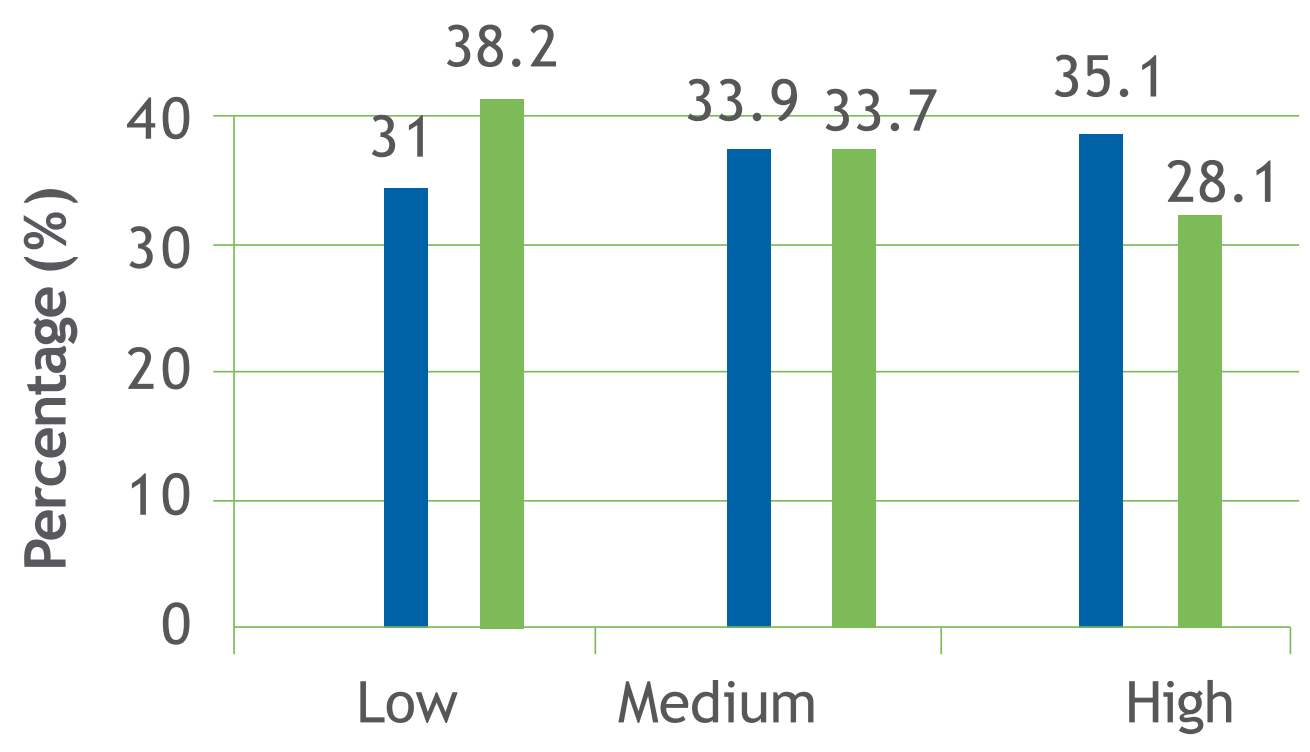

Adherence Score $\quad$ Calcium Vitamin D
- Around third of patients in both groups showed low adherence score; $31 \%$ $(53 / 171), 38.2 \%(128 / 335)$ in calcium and vitamin $D$ groups, respectively (Figure 2).

- Overall, there was significant difference in adherence score betweenage groups $(\mathrm{p}=0.001)$.

- Low adherence score was mostly reported in young age group (18-39 years) for both medications.

- Comparing the adherence score with education level retrieved nostatistical significant difference $(p=0.696)$.

- There was no statistical significant difference among the reasons of non adherence between the two groups (Figure2).

- Forget to take medication was the most reported reason of non adherence in both groups $(29.5 \%, 89 / 302)$.

- Quarter of patients stated that multi-reasons contributed to their non adherence $(24.8 \%, 75 / 302)$.

\section{Conclusion}

Low adherence was high among both calcium and vitamin D groups (around third of both groups), however; there was no significant difference in medications adherence between the two groups. Forget to take medication was the main reason for non adherence.

This study shows the current adherence status among such patients towards their therapy, and promotes further studies to be done in order to improve patient's adherence and eventually their health outcome

Disclosure: None of the authors of this study have to disclose concerning possible financial or personal relationships with commercial entities that may have a direct or indirect interest in the subject matter of this study. This search has been done with help of Medical Research Center-HMC Email: zjassim@hmc.org.qa

\section{Figure 2. Percentage of Non-adherence} Reasons for Calcium \& Vitamin D

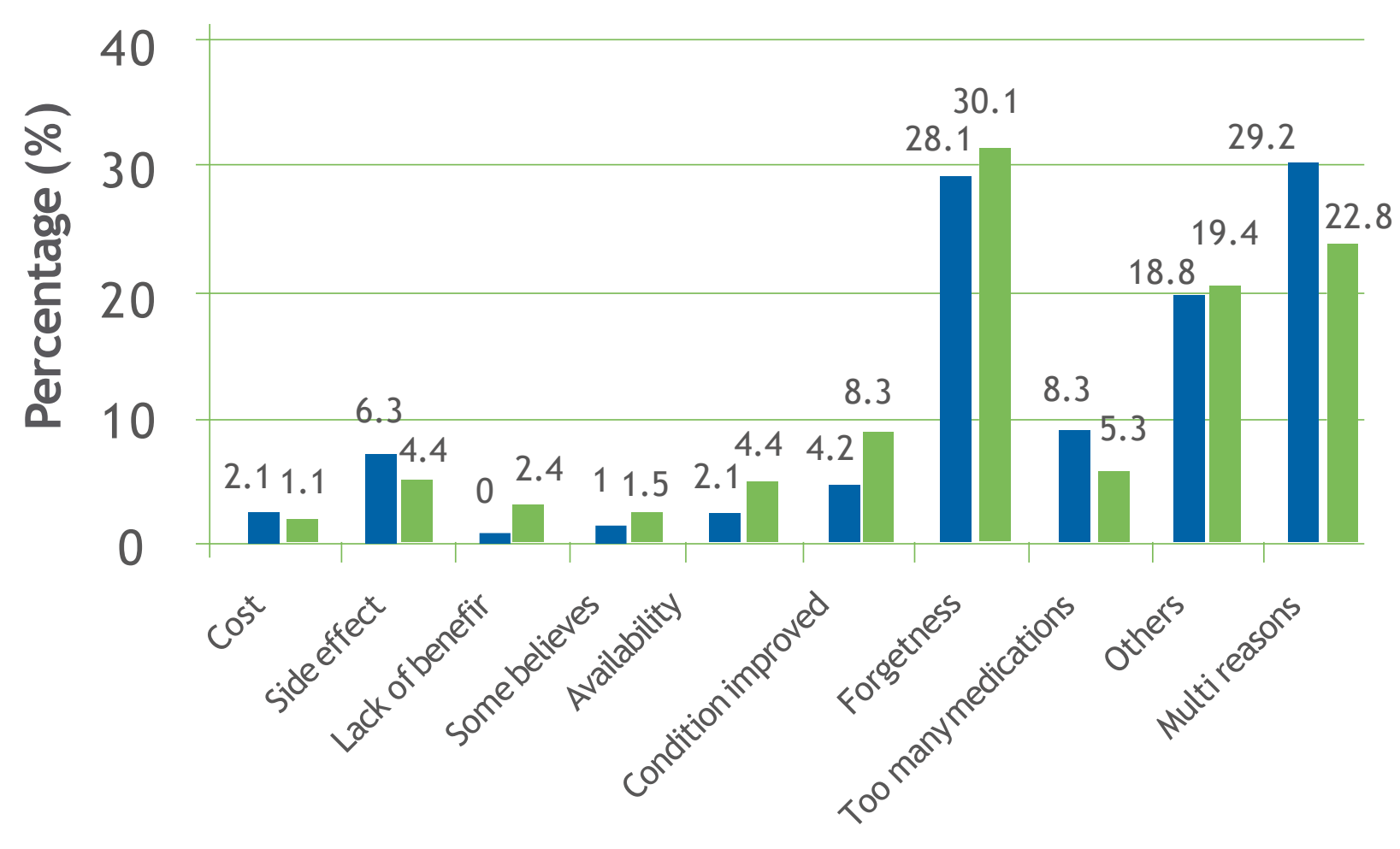

Reasons of non adherence Calcium Vitamin D

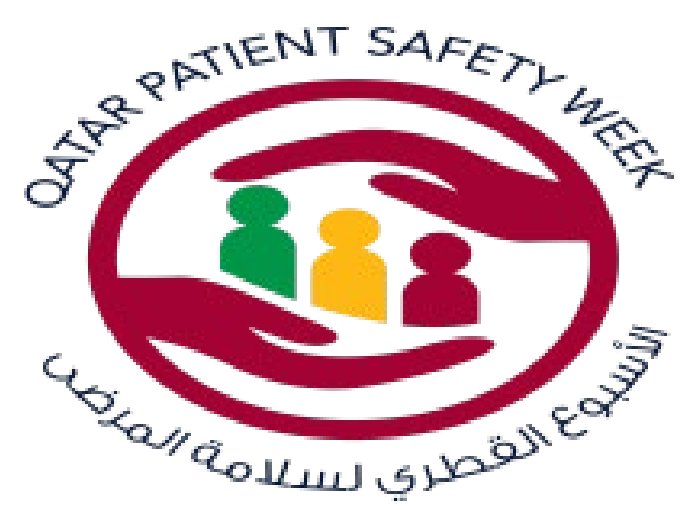

